\title{
CRACK LOCALIZATION IN HYDRAULIC TURBINE BLADES BASED ON KERNEL INDEPENDENT COMPONENT ANALYSIS AND WAVELET NEURAL NETWORK
}

\author{
XIANGHONG WANG* \\ College of Automobile and Mechanical Engineering, Changsha University of Science and Technology, Changsha, \\ Hunan 410004, PR China \\ HANLING MAO \\ College of Mechanical Engineering, Guangxi University, Nanning \\ Guangxi 530003, PR China \\ E-mail:maohl79@gxu.edu.cn \\ www.gxu.edu.cn \\ HONGWEI HU \\ College of Automobile and Mechanical Engineering, Changsha University of Science and Technology, Changsha, \\ Hunan 410004, PR China \\ E-mail: joyhhw@gmail.com \\ www.csust.edu.cn \\ ZHIYONG ZHANG \\ College of Automobile and Mechanical Engineering, Changsha University of Science and Technology, Changsha, \\ Hunan 410004, PR China \\ E-mail: zzy04@163.com \\ www.csust.edu.cn \\ Received 16 November 2012 \\ Accepted 15 April 2013
}

\begin{abstract}
Hydraulic turbine runner has a complex structure, and traditional location methods can't meet its requirement. This paper describes a source location of cracks in turbine blades by combining kernel independent component analysis (KICA) with wavelet neural network (WNN). The research shows that the location accuracy of WNN combined with KICA feature extraction is the best comparing with the results of WNN and back propagation neural network (BPNN). The method decreases the dimension of input parameters and improves the accuracy of location as well.
\end{abstract}

Keywords: Crack localization; acoustic emission (AE); kernel independent component analysis (KICA); scaled conjugate gradient algorithm; wavelet neural network (WNN)

\section{Introduction}

Almost all of hydraulic turbine blades crack after putting into operation. Cracks are seriously dangerous for operating stability and safety of power station. At present, vibration diagnosis is still a widely used method for detecting blade cracks. ${ }^{1}$ The method is an indirect measurement that has a lot of misdiagnosis. It is

\footnotetext{
* Corresponding author: 960, 2nd Section, Wanjiali South RD, College of Automobile and Mechanical Engineering, Changsha University of Science and Technology, Changsha, Hunan, P.R.China.
} 
of great significance to develop a direct nondestructive testing (NDT) method for this kind of operating condition and to find the crack locations.

Acoustic emission (AE) phenomenon is coupled with initiation and propagation of material cracks. ${ }^{2} \mathrm{AE}$ technique by detecting the phenomenon has become a passive dynamic NDT method, which has been widely used to monitor running state of all kinds of equipment. ${ }^{3,4}$ One of its fundamental purposes is to locate crack sources. Location accuracy reflects the conformity between detecting results and really active defects and directly reveals effectiveness of detection method.

In order to monitor the state of the turbine runner and to locate crack sources using AE technique, it needs multi-channel acquisition because of its large scale and complex structure. Thus, the received information is very huge and mutually dependent. In light of this, it is necessary to remove the redundancy and reduce the dimensionality of the received parameters, which can improve the accuracy rate of source location.

Independent component analysis (ICA) is a signal decomposition technique based on higher-order statistic information, rather than the second-order information of sample covariance as used in principal component analysis (PCA). ${ }^{5}$ It can transform a multivariate random signal into a signal with mutually independent components. ICA is suitable for dealing with linear problems because it is a linear transform of observed data. ${ }^{6}$ However, it is possible that strongly comprehensive feature parameters can't be obtained and that useful information can be lost as well, when feature parameters are extracted from essentially nonlinear $\mathrm{AE}$ parameters by ICA. As a result, KICA with nonlinear performance is applied gradually. ${ }^{7,8}$

AE source location methods ${ }^{9,10}$, such as time of arrival (TOA) location, energy location (EL) and modal analysis location (MAL), are to find spatial locations of crack sources according to the information on sensor location or time of sources occurred. These methods are unsuitable for the runner with complex structure. With the development of computer and artificial intelligence technology, artificial neural network (ANN) is used widely. ${ }^{11-13}$ Recently, intelligent location methods, such as ANN, have been developed for the situation of material anisotropy and complex structures. Kirikera et al. ${ }^{14}$ used a passive structural neural system (SNS) for damage localization. The simulation and the experiments show that the SNS was able to localize simulated damages. Kosel et al. ${ }^{15}$ studied an intelligent AE source locator which comprises a sensor antenna and a general regression neural network. The result shows that the intelligent locator can successfully replace the conventional AE locator. The information of ANN is distributed in connection weights, which makes ANN have high fault tolerance and robustness. It realizes source location by nonlinearly mapping feature parameters of input signals into recognition space. However, the general BPNN has local minima points, which makes the location accuracy is difficult to improve. Thus, the support vector machine (SVM) was used. Wang et al. ${ }^{16}$ used SVM to locate crack of turbine runners. The result shows that the recognition rate in the crack region is good with small samples. But SVM has defects for the choice of kernel function and handling of multiple patterns.

WNN is a combination of ANN and wavelet technique. The transfer function of the hidden layer nodes in WNN is a wavelet basis instead of conventional nonlinear transfer function. Thus, the WNN has present resolution in time and frequency domains and also has good ability of function approximation and pattern recognition. Furthermore, WNN is easy to deal with multiple problems and can easily accommodate a variety of prior knowledge, which make WNN be more in line with the requirement of practical problems.

This paper examines crack localization on a real turbine runner from a 302.5MW turbine unit. Two blades are studied, and three AE channels are used. KICA is used to extract feature parameters of crack signals for decreasing the dimension and the redundancy of original $\mathrm{AE}$ parameters (thirty-nine parameters for each signal). WNN is used to locate cracks in runner blades according to the feature parameters aimed at the fact that cracks happen on some special regions in runner. Furthermore, the scaled conjugate gradient algorithm (SCGA) is used in learning iterations of WNN. The results are compared with those based on ICA feature extraction and original AE parameters.

Compared with the Ref.[16], the different methods and different number of samples were used to solve the damage localization in turbine blades. The motivation of this research is to verify the validity of crack location based on KICA and WNN and to find out which is more suitable for the large-size complex structure with a large 
input dimensions and output patterns the large number of samples were used in this study.

\section{KICA Feature Extraction}

The idea of KICA is to map the original input vector $\mathbf{x}_{i}$ ( $k$ dimensions) into a high-dimensional implicit vector feature space $\Phi\left(\mathbf{x}_{i}\right)$ through a function $\Phi$ and then to make components as independent of each other as possible in $\Phi\left(\mathbf{x}_{i}\right)$.

The KICA algorithm for feature extraction has two steps. The first one is to reserve some principal components (PCs) with certain percentage variation of eigenvalues in the feature space and then to pre-whiten the reserved vector so that the whitened variable has a correlation matrix of unity one. The element of the kernel matrix $K_{i j}=k\left(\mathbf{x}_{i}, \mathbf{x}_{j}\right)$ according to radial basis function kernel $K\left(x_{i}, x_{j}\right)=\exp \left(-\frac{\left\|x_{i}-x_{j}\right\|^{2}}{\sigma^{2}}\right)$, where $i, j=1, \ldots, l, l$ is the number of sample and $\sigma$ is the width parameter of the function. The whitened input vector in feature space is $\tilde{\mathbf{x}}_{i}=\left(\sum_{i=1}^{l} \boldsymbol{\alpha}_{i}^{j} k\left(\mathbf{x}_{i}, \mathbf{x}\right)\right)_{j=1}^{k}$, where $\boldsymbol{\alpha}^{j}=\frac{1}{\sqrt{\gamma_{j}}} \mathbf{t}_{j}, j=1, \ldots, k \quad\left(\mathbf{T}=\left[\mathbf{t}_{1}, \ldots, \mathbf{t}_{l}\right]\right.$ and $\mathbf{e}=\left[\gamma_{1}, \ldots, \gamma_{l}\right]$ are the eigenvectors and the eigenvalues of the centered kernel matrix $\mathbf{K}$, respectively).

However, in order to keep $\sum_{i=1}^{l} \Phi\left(\mathbf{x}_{i}\right)=0$ during the whitened process, the kernel matrix $\mathbf{K}$ must be revised as follows,

$$
\mathbf{K}=\mathbf{K}-\frac{1}{l} \mathbf{J J}^{\prime} \mathbf{K}-\frac{1}{l} \mathbf{K J J} \mathbf{J}^{\prime}+\frac{1}{l^{2}}\left(\mathbf{J}^{\prime} \mathbf{K J}\right) \mathbf{J J} \mathbf{J}^{\prime}
$$

where $\mathbf{J}$ is the vector whose elements are the ones with length $l$ and $\mathbf{J}$ is the transpose of $\mathbf{J}$.

The second step is to extract independent components (ICs) by employing ICA for the whitened reserved vector.

ICA is an optimization calculation under some independent criterion. At present, many algorithms have been developed for performing ICA. ${ }^{17-19}$ A very simple and highly efficient fixed-point-FastICA algorithm ${ }^{20}$ is used in the research.

\section{WNN Theory and Algorithm}

The transfer function of hidden layer nodes in the feedforward network is replaced by continuous wavelet function because the wavelet bases have the ability to approximate any function in $L^{2}(R)$. The minimization theory of error function is used, and the waveform and scale of wavelet bases and network weights are adjusted by SCGA ${ }^{21}$ in the research.

As for a three-layer WNN (see Fig.1), given the number of input units is $M$, and the $p$ th input is $X^{p}=\left\{x_{k}^{p}\right\}, k=1,2, \ldots, M$. The number of output units is $L$ and the output value is $Y^{p}=\left\{y_{i}^{p}\right\}, i=1,2, \ldots, L$. The corresponding target output is $D^{p}=\left\{d_{i}^{p}\right\}, p=1,2, \ldots, l$ (where $l$ is the number of all samples). The connection between the $k$ th input unit and the jth hidden unit is called weight $w_{j k}$ and that between the $j$ th hidden unit and the ith output unit is called $w_{i j}$. The number of hidden units is $N(j=1,2, \ldots, N)$. Then the model of WNN is,

$$
y_{i}^{p}=f\left(\sum_{j=1}^{N} w_{i j} \Psi_{a, b}\left(\left(\sum_{k=1}^{M} w_{j k} x_{k}^{p}-b_{j}\right) / a_{j}\right)\right)
$$

where

$$
\Psi_{a, b}\left(n e t_{j}\right)=\cos \left(1.75 n e t_{j}\right) e^{-n e t_{j}^{2} / 2}
$$

$f\left(n e t_{i}\right)=\frac{1}{1+e^{-n e t_{i}}}, a_{j}$ is the wavelet scale factor, and $b_{j}$ is the wavelet location factor. The error function is taken as,

$$
E=\sum_{p=1}^{l} \sum_{i=1}^{L}\left(d_{i}^{p}-y_{i}^{p}\right)^{2}
$$

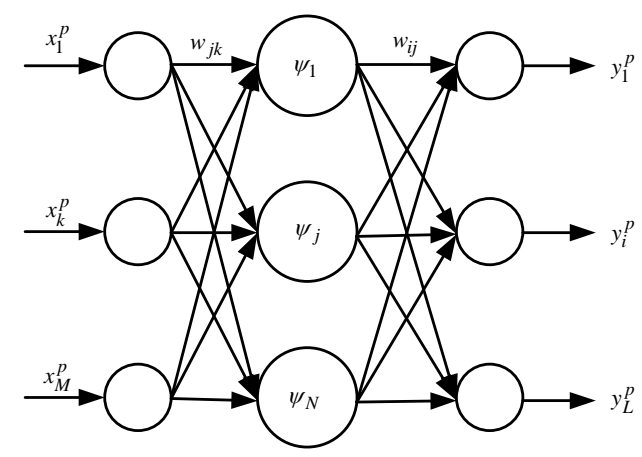

Fig. 1. A schematic of WNN

The SCGA has the advantages of fast descending speed and relatively small memory space. It can obtain good performance in large structural network. The SCGA is mainly to search for a direction $\mathbf{p}_{k}$ and a step size $\alpha_{k}$ in $\mathbf{v}_{k+1}=\mathbf{v}_{k}+\alpha_{k} \mathbf{p}_{k}$. The search direction is, 


$$
\mathbf{p}_{k}=\left\{\begin{array}{cc}
-\mathbf{r}_{k} & k=1 \\
-\mathbf{r}_{k}+\beta_{k-1} \mathbf{p}_{k-1} & k \neq 1
\end{array}\right.
$$

where $\beta_{k}$ is derived from Hestenes-Stiefel formula, i.e. $\beta_{k}=\frac{\Delta \mathbf{r}_{k}^{T} \mathbf{r}_{k+1}}{\Delta \mathbf{r}_{k}^{T} \mathbf{p}_{k}}, \Delta \mathbf{r}_{k}=\mathbf{r}_{k+1}-\mathbf{r}_{k}$ and $\mathbf{r}_{k}=\left.E^{\prime}(\mathbf{v})\right|_{\mathbf{v}=\mathbf{v}_{k}}$. For WNN, v represents four kinds of variables, $a_{j}, b_{j}, w_{i j}$ and $w_{j k}$, and $E^{\prime}(\mathbf{v})$ is the partial derivative of the error function, which is described as,

$$
\left.\begin{array}{c}
\frac{\partial E}{\partial w_{i j}}=-\sum_{p=1}^{l}\left(d_{i}^{p}-y_{i}^{p}\right) f^{\prime}\left(\text { net }_{i}\right) \psi_{a, b}\left(\text { net }_{j}^{p}\right) \\
\frac{\partial E}{\partial w_{j k}}=-\frac{1}{a_{j}} \sum_{p=1}^{l} \sum_{i=1}^{L}\left(d_{i}^{p}-y_{i}^{p}\right) w_{i j} f^{\prime}\left(\text { net }_{i}\right) \psi_{a, b}{ }^{\prime}\left(\text { net }_{j}^{p}\right) x_{k}^{p} \\
\frac{\partial E}{\partial b_{j}}=\frac{1}{a_{j}} \sum_{p=1}^{l} \sum_{i=1}^{L}\left(d_{i}^{p}-y_{i}^{p}\right) f^{\prime}\left(\text { net }_{i}\right) w_{i j} \psi_{a, b}{ }^{\prime}\left(\text { net }_{j}^{p}\right) \\
\frac{\partial E}{\partial a_{j}}=\frac{1}{a_{j}} \sum_{p=1}^{l} \sum_{i=1}^{L}\left(d_{i}^{p}-y_{i}^{p}\right) f^{\prime}\left(\text { net }_{i}\right) w_{i j} \psi_{a, b}\left(\text { net }_{j}^{p}\right) \frac{\text { net }_{j}^{p}-b_{j}}{a_{j}}
\end{array}\right\}
$$

is added to the denominator of $\alpha_{k}$ in order to keep $\mathbf{A}_{k}$ as a positive definite matrix, and $\mathbf{A}_{k}$ is estimated by

$$
\mathbf{A}_{k} \approx \frac{E^{\prime}\left(\mathbf{v}_{k}+\sigma_{k} \mathbf{p}_{k}\right)-E^{\prime}\left(\mathbf{v}_{k}\right)}{\sigma_{k} \mathbf{p}_{k}}, 0<\sigma_{k}<<1 \text {. }
$$

The network learning procedures are presented below,

Step 1: Initiate the network. Set maximum iterative step max_epoch and expected error err_goal. Set scalars epoch $=0, \sigma(>0), \lambda_{k}(>0), \bar{\lambda}_{k}(=0)$ and success=true. Initiate $a_{j}, b_{j}, w_{i j}$ and $w_{j k}$. Then calculate error function $E$ and $\mathbf{r}_{1}=-\mathbf{p}_{1}=\mathrm{E}^{\prime}\left(\mathbf{v}_{1}\right)$.

Step 2: If success=true, then calculate second order information: $\sigma_{k}=\frac{\sigma}{\left|\mathbf{p}_{k}\right|}$ and $\delta_{k}=\mathbf{p}_{k}^{T} \mathbf{A}_{k} \mathbf{p}_{k}$.

Step 3: Calculate $\delta_{k}=\delta_{k}+\left(\lambda_{k}-\bar{\lambda}_{k}\right)\left|\mathbf{p}_{k}\right|^{2}$.

Step 4: If $\delta_{k} \leq 0$, then make the Hessian matrix positive definite: $\quad \bar{\lambda}_{k}=2\left(\lambda_{k}-\frac{\delta_{k}}{\left|\mathbf{p}_{k}\right|^{2}}\right)$, $\delta_{k}=-\delta_{k}+\lambda_{k}\left|\mathbf{p}_{k}\right|^{2}$ and $\lambda_{k}=\bar{\lambda}_{k}$.

Step 5: Calculate step size $\alpha_{k}=\frac{-\mathbf{r}_{k}^{T} \mathbf{p}_{k}}{\delta_{k}}$.

Step 6: Calculate the comparison parameter: $\Delta_{k}=\frac{E(\mathbf{v})-E\left(\mathbf{v}+\alpha_{k} \mathbf{p}_{k}\right)}{E(\mathbf{v})-E\left(\alpha_{k} \mathbf{p}_{k}\right)}$. If $\Delta_{k} \geq 0$, then a successful reduction in error can be made: $\mathbf{v}_{k+1}=\mathbf{v}_{k}+\alpha_{k} \mathbf{p}_{k}$, $\mathbf{r}_{k+1}=\left.E^{\prime}(\mathbf{v})\right|_{\mathbf{v}=\mathbf{v}_{k+1}} \quad, \quad \beta_{k}=\frac{\Delta \mathbf{r}_{k}^{T} \mathbf{r}_{k+1}}{\Delta \mathbf{r}_{k}^{T} \mathbf{p}_{k}}$, $\mathbf{p}_{k+1}=-\mathbf{r}_{k+1}+\beta_{k} \mathbf{p}_{k}$ and set $\bar{\lambda}_{k}=0$ and success $=$ true.

Step 7: If $\Delta_{k} \geq 0.75$, then reduce the scale parameter $\lambda_{k}=0.5 \lambda_{k}$. If $\Delta_{k}<0.25$, then increase the scale parameter $\lambda_{k}=4 \lambda_{k}$.

Step 8: Calculate error $E$ and epoch=epoch +1 . If $E \leq e r r_{-}$goal or epoch = max_epoch , then terminate iterative and return $\mathbf{v}$ as the desired minimum else return to step 2 .

\section{Crack Location in Turbine Blades}

The source location system of turbine runner consists of data acquisition and preprocessing, feature extraction and crack location. It can be summarized as follows:(i) Acquisition of $\mathrm{AE}$ parameters. Crack signals from blades are obtained by AE sensors. AE parameters are picked up and saved by SAMOS system. (ii) AE feature extraction. KICA is used to get rid of redundant information among $\mathrm{AE}$ parameters and only some eigenvalues of the centered kernel matrix are reserved to reduce the dimensionality. (iii) Recognition of crack position. Classification and regression based on WNN are used to locate crack position.

(i) Acquisition of AE parameter

A series of experiments were carried out on an HLA286a-LJ-800 Francis's turbine runner. The schematic of the Francis turbine unit is shown in Fig. 2. Its maximum diameter is $8.6 \mathrm{~m}$, and its height is $5.19 \mathrm{~m}$. The number of runner blades is 13 , and the runner material is stainless steel.

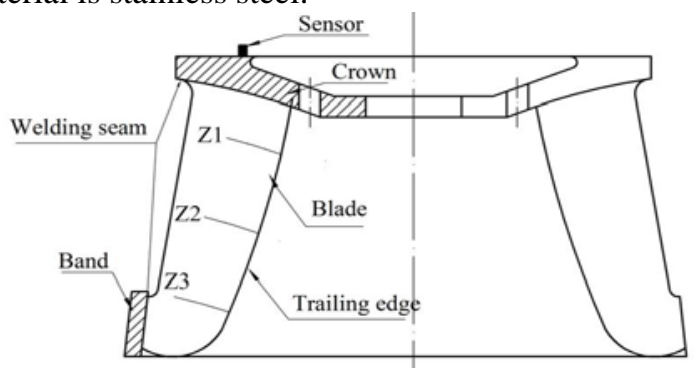

Fig. 2. A schematic of turbine runner

The AE parameters and waveforms were recorded via Physical Acoustics Corporation (PAC) SAMOS system. The PAC R6-a AE sensors (35-100 kHz) and model 2/4/6 pre-amplifiers (10 kHz- $2 \mathrm{MHz})$ were used. 
Signal conditioning was performed by the preamplifiers. The conditioned signal (with a gain of $40 \mathrm{~dB}$ ) was fed to the main data-acquisition board of SAMOS system in which AE waveforms and parameters were stored.

Two blades were tested, and three sensors were mounted on the top of the crown. The surfaces of the crown mounted onto the sensors were polished and cleaned. The interfaces between the sensors and the crown were filled with Vaseline in order to guarantee the performance of signal transmission.

The test undertaken here involved pressing pencil lead, $0.5 \mathrm{~mm} 2 \mathrm{H}$, obliquely against the blade surface until fracture, which is generated simply and repeatedly and has been widely used in researches instead of real signals. $^{22-24}$

Taking account of the cracks of turbine blades were concentrated $^{25}$, three regions of each blade were chosen to locate: the trailing edge of a blade close to the crown, the position in the middle of blade and the trailing edge of a blade close to the band (see Z1-Z3 in Fig. 2). One hundred and two event records were acquired at each region in the two blades. That is, six hundred and twelve samples were used in this study. The total thirty-nine AE features (thirteen parameters per channel, three channels) are calculated including time domain and frequency domain in each sample. The thirteen parameters are rise time, count, energy, duration, amplitude, average frequency, counts to peak, reverberation frequency, initiation frequency, signal strength, absolute energy, frequency centroid and peak frequency, which are extracted by SAMOS system and represent the main information of signal. The test-rig used on site is shown in Fig. 3.

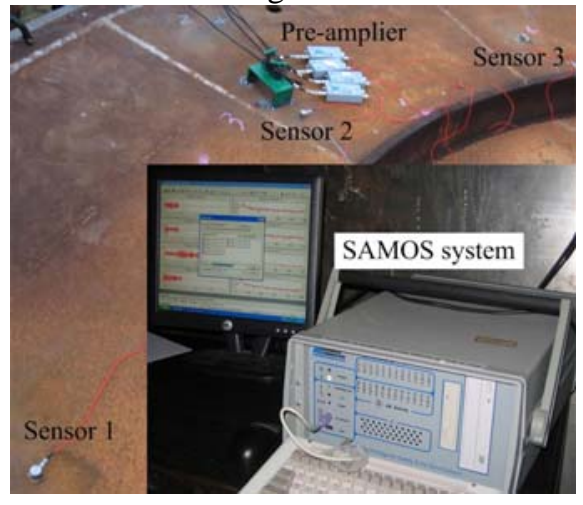

Fig. 3 AE acquisition system employed on-site

(ii) Feature extraction
In order to adequately excavate the effective information in the original AE features and to decrease the effects of different dimension data, all the parameters were normalized by Eq.(6)

$$
P_{i}=\frac{x_{i}-\bar{x}}{\left(\frac{1}{n} \sum_{i=1}^{n}\left(x_{i}-\bar{x}\right)^{2}\right)^{1 / 2}}
$$

where $\bar{x}=\frac{1}{n} \sum_{i=1}^{n} x_{i}$ and $n$ is the number of sample.

For KICA, the normalized parameters were used to form the sample kernel matrix $\mathbf{K}$ according to Eq.(1). The eigenvalues of the matrix $\mathbf{K}$ are in descending order, and the corresponding contribution is also in descending order. The distribution of the first twenty eigenvalues of the matrix is shown in Fig. 4. It is found that the result of feature extraction is better when the radial basis function parameter $\sigma^{2}$ is equal to 2100 by trial and error. At this point, the percentage of the first five eigenvalues and nine eigenvalues of centering kernel matrix is $78.17 \%$ and $89.87 \%$, respectively.

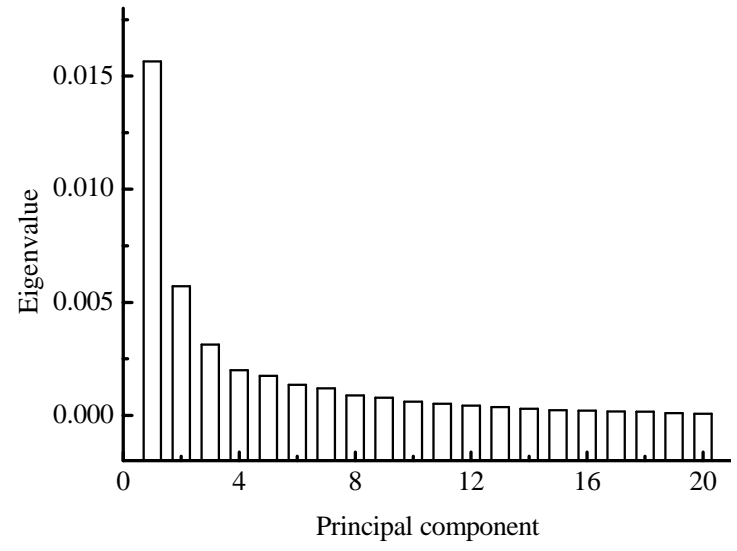

Fig. 4 The first twenty eigenvalues of kernel matrix

At the same time, for ICA feature extraction, the element of the covariance matrix composed by the normalized parameters is $c_{i, j}=\operatorname{cov}\left(x_{i}, x_{j}\right)$.The percentage of the first five eigenvalues and nine eigenvalues of the covariance matrix is $79.85 \%$ and $91.32 \%$, respectively. Representation of the first twenty eigenvalues of the covariance matrix is shown in Fig. 5.

(iii) Results and discussions of crack localization The number of input units of network was determined along with the number of chosen input parameters. The original five parameters are the rise time, count, energy, duration and amplitude from sensor 1. For ICA, the five and nine parameters are the vectors corresponding to the 
first five and nine eigenvalues in the covariance matrix, respectively. And for KICA, the five and nine parameters are also the vectors corresponding to the first five and nine eigenvalues in the kernel matrix, respectively.

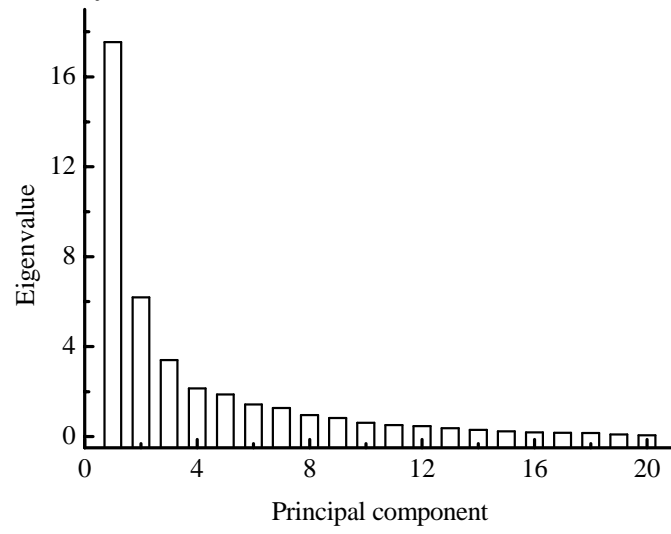

Fig. 5 The first twenty eigenvalues of covariance matrix

The location includes the recognition of crack region and the prediction of distance from crack source to welding seam. There are six output units for the two testing blades (see table 1). The first unit represents the status of blade 1 . The second unit represents the status of blade 2. The third, fourth, fifth unit represent the status of blade regions Z1, Z2, Z3, respectively. And the last one represents the distance from crack source to welding seam normalized by $Y_{i}=\frac{y_{i}-y_{\min }}{y_{\max }-y_{\min }}$.The output value of the first five units is 1 or 0 (1 represents "with crack", 0 represents "without crack") and that of unit 6 is any value between 0 and 1 . In order to recognize the result conveniently, the outputs of blade and crack region were dealt with competing mode, respectively, that was, only maximum value was set to 1 and the else is set to 0 .

The number of hidden units was chosen in $[3,17]$ according to the empirical formula $n=\sqrt{L+M}+a$ (where $\mathrm{L}$ is the number of output units, $\mathrm{M}$ is the number of input units and a is a constant in $[0,10])$, and the value with the best result was taken (see table 3 ). For the six hundred and twelve samples, nineteen samples from each crack region were used as testing samples, the remainders were training samples. The target outputs of the testing samples are shown in table 2. The iterative steps were set to 2000, and the expected error was 0.001 . At the same time, the results were compared with those obtained by BPNN. The transfer functions of both the hidden layer nodes and the output layer nodes in BPNN were $f(n e t)=\frac{1}{1+e^{-n e t}}$. The other setups were the same with WNN.

Table 1. Output unit

\begin{tabular}{ccc}
\hline No. & Content & Value \\
\hline 1 & Blade 1 & 0 or 1 \\
2 & Blade 2 & 0 or 1 \\
3 & Region close to crown & 0 or 1 \\
4 & Region in the middle of blade & 0 or 1 \\
5 & Region close to band & 0 or 1 \\
6 & Normalization distance & {$[0,1]$} \\
\hline
\end{tabular}

Table 2. Target values of testing samples

\begin{tabular}{|c|c|}
\hline No. & Target value \\
\hline 1 & $\left(\begin{array}{llllll}1 & 0 & 1 & 0 & 0 & 0.025\end{array}\right)$ \\
\hline 2 & $\left(\begin{array}{lllllll}1 & 0 & 1 & 0 & 0 & 0\end{array}\right)$ \\
\hline 3 & $\left(\begin{array}{l}1 \\
0\end{array} 00100.639\right)$ \\
\hline 4 & 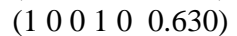 \\
\hline 5 & 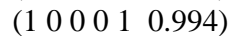 \\
\hline 6 & $\left(\begin{array}{lllllll}1 & 0 & 0 & 0 & 1 & 1\end{array}\right)$ \\
\hline 7 & $\left(\begin{array}{llllll}0 & 1 & 0 & 0 & 1 & 0.994\end{array}\right)$ \\
\hline 8 & $\left(\begin{array}{lllllll}0 & 1 & 0 & 0 & 1 & 1\end{array}\right)$ \\
\hline 9 & $\left(\begin{array}{llllll}0 & 1 & 0 & 1 & 0 & 0.633\end{array}\right)$ \\
\hline$\ldots$ & \\
\hline 106 & $\left(\begin{array}{lllllll}0 & 1 & 0 & 1 & 0 & 0.620\end{array}\right)$ \\
\hline 107 & $\left(\begin{array}{lllllll}0 & 1 & 1 & 0 & 0 & 0.013\end{array}\right)$ \\
\hline 108 & $\left(\begin{array}{lllllll}0 & 1 & 1 & 0 & 0 & 0.025\end{array}\right)$ \\
\hline
\end{tabular}

Tables 3 and 4 display the results and the errors of WNN and BPNN with different input parameters, respectively. It shows that the result of region recognition using nine feature parameters extracted by KICA is the best in the two types of networks. Its accuracy rate is $100 \%$. The worst one is to input five original AE parameters for BPNN, 79.82\%. The recognition rates of WNN are better than the corresponding results of BPNN. The recognition errors mainly exist in the blade regions (i.e. output units 3 5), which is because the transmission speed of AE signal in stainless steel is slow so that the difference of $\mathrm{AE}$ parameters from different blade regions is small. In addition, the results based on KICA feature parameters are better than those based on ICA due to good quality of data input after nonlinear feature extraction.

For the prediction distances from $\mathrm{AE}$ sources to welding seam, the mean square error (MSE) of WNN is less than that of BPNN. The minimum MSE is also obtained by nine KICA feature parameters, followed by five KICA feature parameters. The worst one is from the five original parameters. Likewise, the prediction distance of WNN with nine KICA feature parameters is 
Table 3. Testing results.

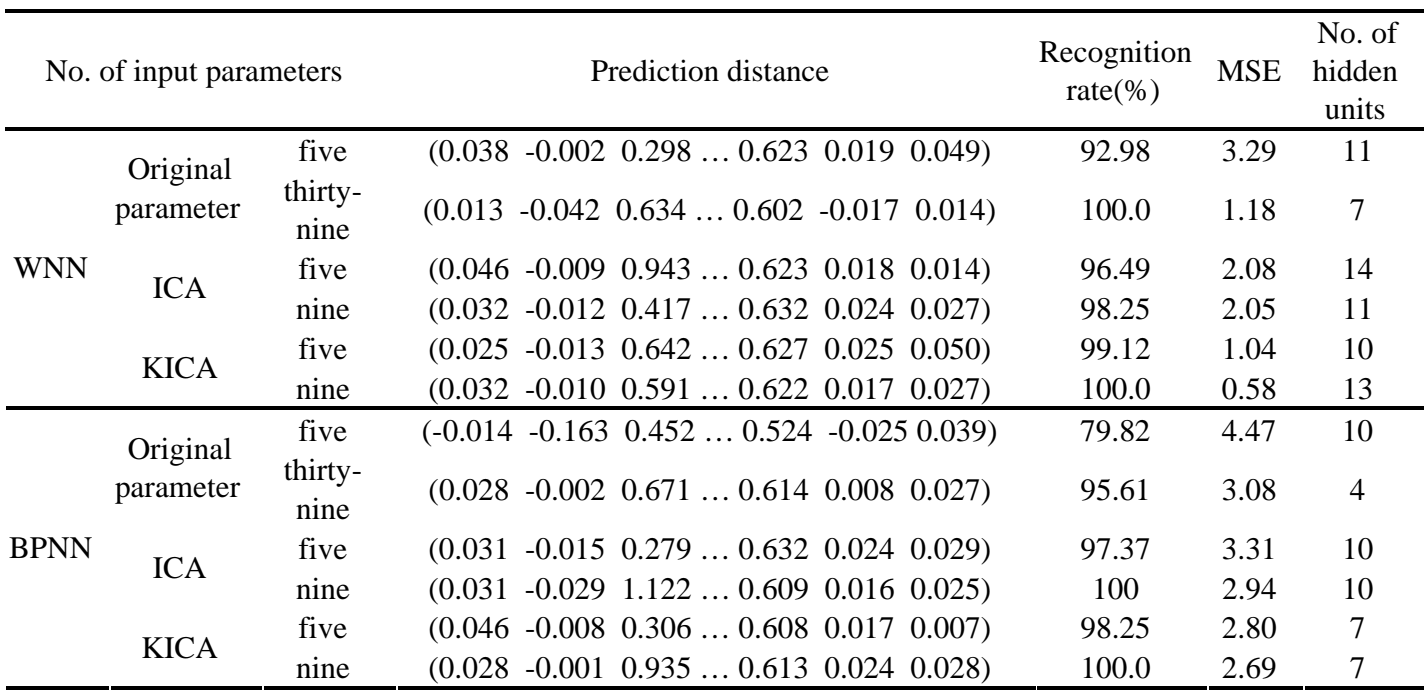

Table 4. Errors of testing results

\begin{tabular}{|c|c|c|c|c|}
\hline \multicolumn{3}{|c|}{ No. of input parameters } & Error of prediction distance & $\begin{array}{c}\text { Error of } \\
\text { Recognition rate(\%) } \\
\end{array}$ \\
\hline \multirow{4}{*}{ Original } & \multirow{2}{*}{ five } & WNN & $(-0.013,0.002,0.341, \ldots,-0.003,-0.006,-0.024)$ & 7.02 \\
\hline & & BPNN & $(0.039,0.163,0.005, \ldots, 0.096,0.038,-0.014)$ & 20.18 \\
\hline & \multirow{2}{*}{$\begin{array}{c}\text { thirty- } \\
\text { nine }\end{array}$} & WNN & $(0.012,0.042,-0.304, \ldots, 0.018,0.030,0.011)$ & 0 \\
\hline & & BPNN & $(-0.003,0.002,0.222, \ldots, 0.006,0.005,-0.002)$ & 4.39 \\
\hline \multirow{4}{*}{ ICA } & \multirow{2}{*}{ five } & WNN & $(-0.021,0.009,-0.003, \ldots,-0.003,-0.005,0.011)$ & 3.51 \\
\hline & & BPNN & $(-0.006,0.015,0.048, \ldots,-0.012-0.011-0.004)$ & 2.63 \\
\hline & \multirow{2}{*}{ nine } & WNN & $(-0.007,0.012,0.189, \ldots,-0.012,-0.011,-0.002)$ & 1.75 \\
\hline & & BPNN & $(-0.006,0.029,-0.032, \ldots, 0.011,-0.003, \quad 0)$ & 0 \\
\hline \multirow{4}{*}{ KICA } & \multirow{2}{*}{ five } & WNN & $(0,0.013,0.360, \ldots,-0.007,-0.012,-0.025)$ & 0.88 \\
\hline & & BPNN & $(-0.021,0.008,-0.483, \ldots, 0.012,-0.004,0.018)$ & 1.75 \\
\hline & \multirow{2}{*}{ nine } & WNN & $(-0.007,0.010,0.333, \ldots,-0.002,-0.004,-0.002)$ & 0 \\
\hline & & BPNN & $(-0.003,0.001,-0.296, \ldots, 0.007,-0.011,-0.003)$ & 0 \\
\hline
\end{tabular}

the best. The maximum error is $34 \mathrm{~cm}$, which is 57.3 percent of the real distance. And the minimum error is almost $0 \mathrm{~cm}$. The average error of the all samples is $2.0 \%$. For BPNN inputting nine KICA feature parameters, the maximum distance error is $-94 \mathrm{~cm}, 58.7$ percent of the real distance. The minimum error is also almost $0 \mathrm{~cm}$. The average error of the testing samples is 5.1 percent. Likewise, the worst prediction result is from five original parameters. Its maximum error is 57 cm (35.6 percent), and the average error rate is 7.5 percent for WNN. The maximum error is $86 \mathrm{~cm}(145.4$ percent), and the average error rate is 19.1 percent for BPNN.
Although the result of using all original parameters is also good, the dimension of input data will be very huge when the whole runner is located by multi-channel, which will affect the location result and propagation of data. In addition, the number of the hidden layer neurons required in the cases of BPNN is less than that required in the cases of WNN, which shows that the structure of BPNN is simpler than that of the WNN. Furthermore, the result of each testing sample from WNN is not always better than that from BPNN because of the effect of initial values of networks, but the average error of the testing samples of WNN is less than that of BPNN. 


\section{Conclusions}

The result of nine KICA feature parameters for WNN is the best. It not only recognizes all the crack regions accurately, but acquires the minimum error range of prediction distance. The worst one is from five original parameters. As a result, in real-world applications, it can decrease the dimension of input parameters by KICA feature extraction, which reduces the burden on data transmission and storage and improves the accuracy of location as well. Furthermore, the location method overcomes particular problems associated with source location in complex structures using some current location techniques (TOA, EL and MAL). It does not require information about sensor location or time of occurrence of source. In light of these, it is a good method for source location in complex large-size structures to combine KICA with WNN. However, the number of training samples increases exponentially

\section{References}

1. G. Wu, W. Liang and H.Wang, Detecting vane cracking of hydraulic turbine with acoustic emission technology, Power System and Clean Energy 25(2009) 58-60 (in Chinese).

2. H. Shaikh, R. Amirthalingam, T. Anita, N. Sivaibharasi, T. Jaykumar, P. Manohar and H. S. Khatak,Evaluation of stress corrosion cracking phenomenon in an AISI type 316LN stainless steel using acoustic emission technique, Corros. Sci. 49(2)(2007)740-765.

3. M. S. H. Bhuiyan, I. A. Choudhury and Y. Nukman, An innovative approach to monitor the chip formation effect on tool state using acoustic emission in turning, Int. J. Mac. Tool. Manufact. 58 (2012) 19-28.

4. B. Eftekharnejdad and D. Mba, Seeded fault detection on helical gears with acoustic emission, Appl. Acoust. 70(4) (2009) 547-555.

5. P. Comon, Independent component analysis, A new concept?, Signal Process. 36(3)(1994)287-314.

6. A. Singer and R. R. Coifman, Non-linear independent component analysis with diffusion maps, Appl. Comput. Harmon. A. 25(2) (2008) 226-239.

7. A. Widodo and B. S. Yang, Application of nonlinear feature extraction and support vector machines for fault diagnosis of induction motors, Expert. Syst. Appl. 33(1) (2007)241-250.

8. Y. Zhang and C. Ma, Decentralized fault diagnosis using multiblock kernel independent component analysis, Chem. Eng. Res. Des. 90(5) (2012)667-676.

9. D. A. Axinte, D.R. Natarajan and N. N. Z. Gindy, An approach to use an array of three acoustic emission sensors to locate uneven events in machining--Part 1 : method and validation, Int. J. Mach. Tool. Manufact. 45(14) (2005)1605-1613. when the number of input parameters (units) is large, which makes the convergence speed of WNN greatly decrease. The next stage of this work will focus on the study of improving the convergence speed so that it will be suitable for a real-world application.

\section{Acknowledgements}

The authors wish to express their gratitude to the Institute of Electricity Experiment and Yantan water power plant, Guangxi province, for their assistance in undertaking this investigation. This research is supported by the National Natural Science Foundation of China (No. 51105045 and No.51205031) and the Scientific Research Fund of Hunan Provincial Education Department (No.10B005).

10. P. Kundu, N. K. Kishore and A. K. Sinha, A non-iterative partial discharge source location method for transformers employing acoustic emission techniques, Appl. Acoust. 70(11-12) (2009)1378-1383.

11. S. Tasdemir, I. Saritas, M. Ciniviz and N. Allahverdi, Artificial neural network and fuzzy expert system comparison for prediction of performance and emission parameters on a gasoline engine, Expert Syst. Appl. 38(11)(2011)13912-13923.

12. V. Baiju and C. Muraleedharan, Artificial neural network modelling of adsorbent bed in a solar adsorption refrigeration system, Proc. IMechE Part C: J. Mech. Eng. Sci. (2012) DOI:0954406212448606.

13. S. Kiranyaz, T. Ince, A. Yildirim and M. Gabbouj, Evolutionary artificial neural networks by multidimensional particle swarm optimization. Neural Networks 22(10) (2009)1448-1462.

14. G. R. Kirikera, V. Shinde, M. J. Schulz, A. Ghoshal, M. Sundaresan and R. Allemang, Damage localisation in composite and metallic structures using a structural neural system and simulated acoustic emissions, Mech. Syst. Signal Process. 21(1)(2007)280-297.

15. T. Kosel, I. Grabec and F. Kosel, Intelligent location of two simultaneously active acoustic emission sources, Aerosp. Sci. Technol. 9(1)(2005) 45-53.

16. X. H. Wang, H. L. Mao, C. M. Zhu and Z. F. Huang, Damage localization in hydraulic turbine blades using kernel independent component analysis and support vector machines, Proc. IMechE Part C: J. Mech. Eng. Sci. 223(2)(2009)2525-2529.

17. S. E. Barriga, M. Pattichis, D. Ts'o, M. Abramoff, R. Kardon, Y. Kwon and P. Soliz, Independent component analysis using prior information for signal detection in a functional imaging system of the retina. Med. Image Anal. 15(1)(2011)35-44. 
18. D. S. Huang and J. X. Mi. A New Constrained Independent Component Analysis Method. IEEE Trans. Neural Netw. 18(5) (2007)1532-1535.

19. Y. Xue, Y. Wang and J. Yang, Independent component analysis based on gradient equation and kernel density estimation. Neurocomputing. 72(7-9) (2009)1597-1604.

20. A. Hyvarinen, Fast and robust fixed-point algorithms for independent component analysis. IEEE Trans. Neural Netw. 10(3)(1999)626-634.

21. M. F. Moller, A scaled conjugate gradient algorithm for fast supervised learning. Neural Networks 6(4)(1993)525-533.

22. P. Nivesrangsan, J. A. Steel and R. L. Reuben, AE mapping of engines for spatially located time series. Mech. Syst. Signal Process. 19(5)(2005)1034-1054.

23. D. Mba and L. D. Hall, The transmission of acoustic emission across large-scale turbine rotors. NDT E Int. 35(8)(2002)529-539.

24. ASTM E976-99. Standard guild for determining the reproducibility of acoustic emission sensor response. American Society for Testing and Materials, 1999,pp. 395-403.

25. S.X. Gu, Cracks in turbine runner and its treatment. Mechanical \& Electrical Technique of Hydropower Station 16(3)(1992)1-7 (In Chinese). 